\title{
Review
}

\section{Systematic studies on the species of Chironomidae recorded from Japan during the period from September 1997 to August 2000}

\author{
Manabu SASA ${ }^{1)}$ and Hiroshi Suzuki ${ }^{2)}$ \\ 1) Kankyo Fukushi Kenkyusho (Institute of Environmental and Welfare Studies) \\ Aramata 135-3, Kurobe-shi, 938-0001 Japan \\ ${ }^{2)}$ Institute of Tropical Medicine, Nagasaki University, Sakamotocho, Nagasaki, \\ 852-8523 Japan
}

(Received: 30 August 2000; Accepted: 31 January 2001)

Key words: Chironomidae, taxonomy, new species, allergen, water quality

\begin{abstract}
The number of species of insect family Chironomidae breeding in Japan is very large, and more than one thousand species have already been recorded from this small country, including many new species. In the Monograph of Chironomidae by Sasa and Kikuchi (1995), a total of 757 species was recorded, among which only 131 were those already recorded from Europe, Americas, and other regions outside of Japan and East Asia, and in another monograph compiled by Sasa (1998), 176 species were added. In the present paper, additional 241 species recorded from Japan in 20 papers published as of July 2000 are recorded by according to taxonomic groups making the total number of Chironomidae of Japan 1,174.
\end{abstract}

\section{INTRODUCTION}

Extensive studies on taxonomy and biology of the insect family Chironomidae were commenced mainly by $\mathrm{M}$. Tokunaga, Kyoto University, who recorded about 160 species from the present territory of Japan during the period from 1932 to 1965. Additional studies on the taxonomy and distribution of Chironomidae of Japan were started after World War II, mainly by H. Hashimoto, Shizuoka University, from about 1957 , by M. Sasa and his coworkers, National Institute of Environmental Studies from about 1977, and by M. Yamamoto, Kyushu University, from about 1980 , and a great deal of new information has been added. In the checklist of Chironomidae of Japan compiled by Sasa (1989), a total of 480 chironomid species had been recorded from Japan, among which only
$131(27.3 \%)$ were considered as belonging to the same species as those already described in other regions of the world including Europe, Africa and America, and the rest $349(72.7 \%)$ were described as new species indigenous to Japan and compiled by Sasa and Kikuchi (1995), 757 species were recorded, and 535 among them were new species recorded by Sasa and coworkers. In the second monograph of "Chironomidae of Japan, 1998" compiled about 3.5 years thereafter by Sasa (1998), 176 species and 19 genera were added, and the total number of species reached 933 at this stage.

As shown in the reference of the present paper, another 20 papers were thereafter published as new records of Chirinomidae of Japan as of July 2000, and a total of 241 species is newly recorded, mostly as new species. In the present paper, they are arranged by taxonomic groups according to 
our present system. They are classified into 55 species and 19 genera of tribe Chirinomini, 58 species and 4 genera of tribe Metriocnemini, 7 species and 2 genera of tribe Corynoneurini, 3 species and 3 genera of subfamily Diamesinae, 2 species and 2 genera of subfamily Podonominae, and 9 species and 6 genera of subfamily Tany podinae, and the total number of species of Chironomidae recorded from the present territory of Japan reached 1,174 as of July 2000. This kind of information is considered necessary for future systematic studies on this family of insects, which seems to have still many more unrecorded species in Japan and other region of the world.

The studies on Chironomidae are important not only in the taxonomy of this group of insects, but also in relation to environmental biology as indicators of water quality, as highly beneficial insects in the natural improvement of waters in lakes and streams, but also in medical entomology as causative agents of allergens such as bronchial asthma.

\section{Materials AND Methods}

Very many species of the non-biting midges, or the insect family Chironomidae (Diptera), have been collected by us from a number of localities in Japan, from the main islands of Hokkaido, Honshu, Shikoku and Kyushu, and also from many adjacent islands, and the number of species reached a total of 1,174 as of July 2000. Their larvae and pupae were collected mostly from various types of stagnant or running waters, but some also from the soil, and were identified mostly after the adult males were obtained by laboratory rearing. However, most of the specimens were collected while swarming in the air or resting in bushes or trees with insect net during the day, or at night with sucking tubes while attracted to light or with light traps.

The adult specimens thus collected were usually preserved in $70 \%$ ethanol solution in glass vials, and were individually dissected and mounted on slides on gumchloral medium after the method described in "The Monograph of Chirinomidae of Japan" by Sasa and Kikuchi (1995) and of Sasa (1998), and were also described in the following references.

\section{A List of Chirinomid species recorded from Japan after 1998}

Scientific name, reference, page and Fig. No.

Subfamily CHIRONOMINAE Tribe Chironomini "The Chironomus complex"

\begin{tabular}{|c|c|c|}
\hline Chironomus sp. "simantoabeus" Sasa, Suzuki et Sakai, 1998a; & p. 49 , & Fig. 1 \\
\hline " simantobeceus & p. 49 , & Fig. 2 \\
\hline tusimaabeus Sasa et Suzuki, 1999a; & p. 2 , & Fig. 1 \\
\hline Endochironomus hibaradecimus Sasa et Suzuki, 1998b; & p. 26, & Fig. B1 \\
\hline Yaesecundus Sasa et Suzuki, 2000a; & p. 12 & \\
\hline Yaesecundus iriobeceus Sasa et Suzuki, 2000a; & p. 12 , & Fig. 11 \\
\hline \multicolumn{3}{|c|}{ "The Harnischia complex" } \\
\hline Cryptochironomus jokaprimus Sasa et Ogata, 1999; & p. 86 , & Fig. 1 \\
\hline Cryptotendipes sibaabeus Sasa, Sumita et Suzuki, 1999; & p. 184 & $\begin{array}{l}\text { Fig. } 3 \\
\text { Fig. } 10\end{array}$ \\
\hline Harnischia ohmuraensis Kobayashi et Suzuki, 1999a; & p. 80 & Fig. 1 \\
\hline " simantocedea Sasa, Suzuki et Sakai, 1998a; & p. 50 , & Fig. 3 \\
\hline sibabecea Sasa, Sumita et Suzuki, 1999; & p. 185 , & Fig. 4 \\
\hline
\end{tabular}


Parachironomus monochromus (van der Wulp, 1874);

in Kobayashi et Suzuki, 1999a;

Paracladopelma misumaiprima Sasa et Suzuki, 1998b;

sp. "misumaisecunda", in Sasa et Suzuki, 1998b;

simantodeea Sasa, Suzuki et Sakai, 1998a;

"The Polypedilum complex"

Microtendipes simantofegeus Sasa, Suzuki et Sakai, 1998a;

simantogeheus " 1998a;

tusimabeceus Sasa et Suzuki, 1999a;

tusimacedeus "

tusimadeeus

iriocedeus Sasa et Suzuki, 2000a;

Pentapedilum jokasecundum Sasa et Ogata, 1999;

tusimaefeum Sasa et Suzuki, 1999a;

tusimafegeum

yakuabeum Sasa et Suzuki, 2000b;

iriodeeum Sasa et Suzuki, 2000a;

The nubifer group of subgenus Polypedilum

Polypedilum (Polypedilum) ginzanprimum Sasa et Suzuki, 1998b;

\section{Polypedilum}

"
"
"
"
"

mismaitertium

simantoheium Sasa, Suzuki et Sakai, 1998a;

simantoijeum " " 1998a;

simantomaculatum " " 1998a;

tusimageheum Sasa et Suzuki, 1999a;

tusimaheium Sasa et Suzuki, 1998a;

yakubeceum Sasa et Suzuki, 2000b;

yakucedeum Sasa et Suzuki, 2000b;

The nubeculosum group of subgenus Polypedilum

Polypedilum (Polypedilum) misumaiquartum Sasa et Suzuki, 1998b;

"
"
"
"

"
"
"
"

tusimaijeum Sasa et Suzuki, 1999a;

yakudeeum Sasa et Suzuki, 2000b;

isigabeceum Sasa et Suzuki, 2000a;

iriofegeum

iriogeheum

* (lateral seta on dorsal appendage is occasionally absent in this species)

The subgenus Tripodura, genus Polypedilum

Polypedilum (Tripodura) ginzansecundum Sasa et Suzuki, 1998b;

$$
\text { " " sibadeeum Sasa, Sumita et Suzuki, 1999; }
$$

The subgenus Uresipedilum, genus Polypedilum

Polypedilum (Uresipedilum) ginzantertium Sasa et Suzuki, 1998b;

$$
\text { " " simantokeleum Sasa, Suzuki et Sakai, 1998a; }
$$

Prochironomus irioheius Sasa et Suzuki, 2000a;

Stenochironomus irioijeus Sasa et Suzuki, 2000a;

" gotoabeus Sasa et Suzuki, 2000c;

" sibaefeus Sasa, Sumita et Suzuki, 1999;

Tosayusurika Sasa, Suzuki et Sakai, 1998a;

Tosayusurika simatoefea Sasa, Suzuki et Sakai, 1998a;

Yaeprimus Sasa et Suzuki, 2000a;

Yaeprimus isigaabeus Sasa et Suzuki, 2000a;

Yaequartus Sasa et Suzuki, 2000a;

Yaequartus iriokeleus Sasa et Suzuki, 2000a;

Yaetertius Sasa et Suzuki, 2000a; p. 185 , Fig. 8

new record to Japan,

p. $82, \quad$ Fig. 2

p. 18, Fig. A10

p. 19, Fig. A11

p. $51, \quad$ Fig. 4

$\begin{array}{ll}\text { p. } 53, & \text { Fig. } 6 \\ \text { p. } 54, & \text { Fig. } 7 \\ \text { p. } 4, & \text { Fig. } 2 \\ \text { p. } 5, & \text { Fig. } 3 \\ \text { p. } 5, & \text { Fig. } 4 \\ \text { p. } 12, & \text { Fig. } 12 \\ \text { p. } 89, & \text { Fig. } 2 \\ \text { p. } 6, & \text { Fig. } 5 \\ \text { p. } 7, & \text { Fig. } 6 \\ \text { p. } 56, & \text { Fig. } 3 \\ \text { p. } 13, & \text { Fig. } 13\end{array}$

p. 13, Fig. A5

p. 20, Fig. A13

p. $56, \quad$ Fig. 9

p. $57, \quad$ Fig. 10

p. $58, \quad$ Fig. 11

p. $8, \quad$ Fig. 7

p. $9, \quad$ Fig. 8

p. $57, \quad$ Fig. 5

p. $57, \quad$ Fig. 6

p. 21, Fig. A14

p. 12, Fig. 13

p. $59, \quad$ Fig. 9

p. $5, \quad$ Fig. 2

p. 15, Fig. 15

p. 16, Fig. 16

p. 14, Fig. A6

p. 187 , Fig. 6

p. 15, Fig. A7

p. 59, Fig. 12

p. 16, Fig. 17

p. $17, \quad$ Fig. 18

p. 142, Fig. 1

p. 188 , Fig. 7

p. 52

p. 52 , Fig. 5

p. 4

p. $4, \quad$ Fig. 1

p. 19

p. 19, Fig. 20

p. 18 
"Tribe Tanytarsini"

Cladotanytarsus simantolemeus Sasa, Suzuki et Sakai, 1998a; tusimajekeus Sasa et Ogata, 1999; yakuefeus Sasa et Suzuki, 2000b; yakufegeus Sasa et Suzuki, 2000b;

Micropsectra jokatertia Sasa et Ogata, 1999;

" jokaquarta Sasa et Ogata, 1999;

" simantoneoa Sasa, Suzuki et Sakai, 1998a;

" tusimakelea Sasa et Suzuki, 1999a;

" tusimalemea

"tusimamenea

tusimanenoa

tusimaopea

tusimapequea

tusimaquerea

tusimaresea

Rheotanytarsus tusimaseteus Sasa et Suzuki, 1999a;

Tanytarsus ginzanquartus Sasa et Suzuki, 1998b;

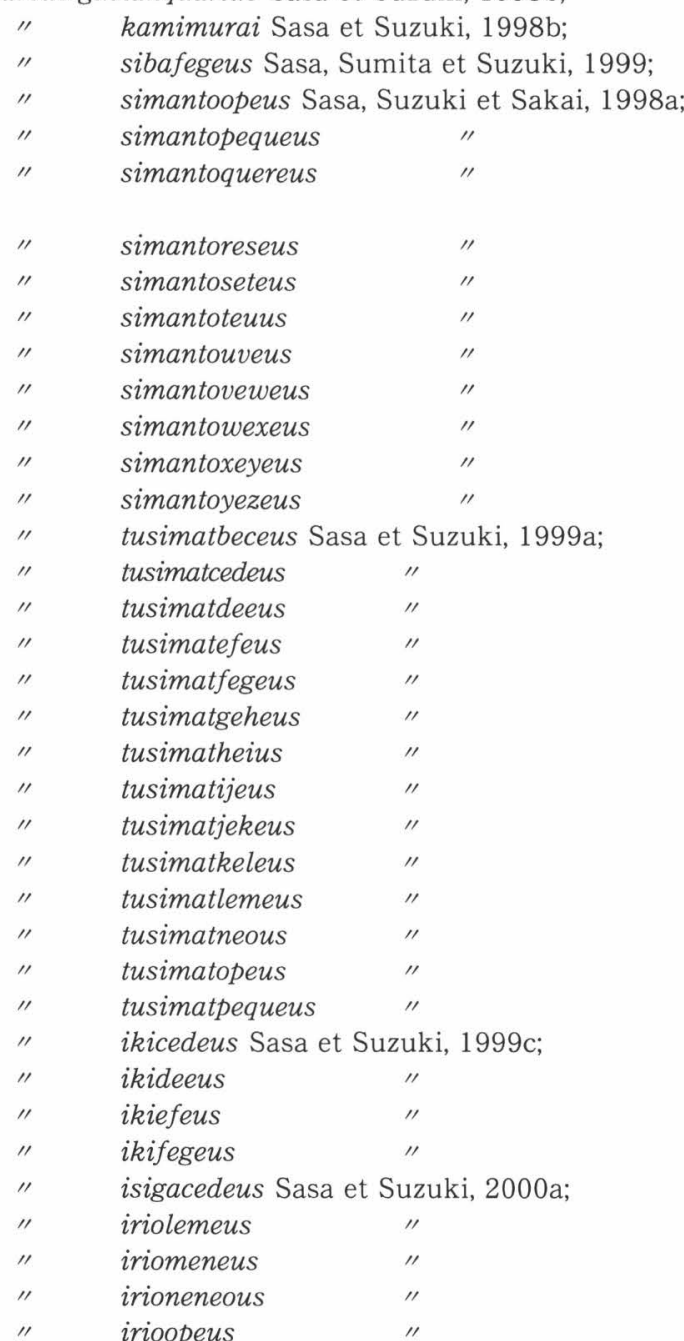

p. 60, Fig. 13

p. 13, Fig. 14

p. 60 , Fig. 12

p. $61, \quad$ Fig. 13

p. $92, \quad$ Fig. 4

p. $93, \quad$ Fig. 5

p. 62, Fig. 15

p. 16, Fig. 16

p. 17, Fig. 18

p. $18, \quad$ Fig. 19

p. 19, Fig. 20

p. 19, Fig. 21

p. 20, Fig. 22

p. 21, Fig. 23

p. 22, Fig. 24

p. 23, Fig. 25

p. 16, Fig. A8

p. 30, Fig. B4

p. $189, \quad$ Fig. 9

p. 63, Fig. 16

p. $63, \quad$ Fig. 17

p. 64, Fig. 18

also in p. $70, \quad$ Fig. 26

p. 65, Fig. 19

p. $66, \quad$ Fig. 20

p. 67, Fig. 21

p. $68, \quad$ Fig. 22

p. 68 , Fig. 23

p. 69 , Fig. 24

p. $70, \quad$ Fig. 25

p. 71 , Fig. 27

p. 25, Fig. 27

p. 25, Fig. 28

p. $26, \quad$ Fig. 29

p. 27, Fig. 30

p. 27, Fig. 31

p. 28, Fig. 32

p. 29, Fig. 33

p. $30, \quad$ Fig. 35

p. 31 , Fig. 36

p. $31, \quad$ Fig. 37

p. $32, \quad$ Fig. 38

p. $33, \quad$ Fig. 40

p. 34, Fig. 41

p. 35, Fig. 42

p. $149, \quad$ Fig. 4

p. $150, \quad$ Fig. 5

p. 150 , Fig. 6

p. $151, \quad$ Fig. 7

p. $6, \quad$ Fig. 3

p. $20, \quad$ Fig. 21

p. 21, Fig. 22

p. 21, Fig. 23

p. 21, Fig. 24 


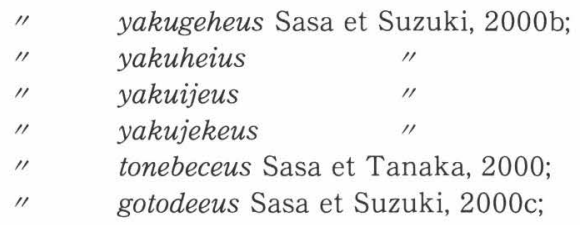

$\begin{array}{ll}\text { p. } 64, & \text { Fig. } 17 \\ \text { p. } 64, & \text { Fig. } 18 \\ \text { p. } 65, & \text { Fig. } 19 \\ \text { p. } 66, & \text { Fig. } 20 \\ \text { p. 1, } & \text { Fig. } 2 \\ \text { p. 152, } & \text { Fig. } 7\end{array}$

Tribe Orthocladiini, Subfamily Orthocladiinae

Kurobebrillia Sasa et Ogata, 1999;

Kurobebrillia jokaquinta Sasa et Ogata, 1999;

Pseudobrillia gotobeca Sasa et Suzuki, 2000c;

$\begin{array}{ccc}\text { " } & \text { tusimoabeus Sasa et Suzuki, 1999b; } \\ \text { " } & \text { (Isocladius) } & \text { uresibeceus Sasa et Suzuki, 1998a; } \\ \text { " } & \text { " } & \text { ikigeheus Sasa et Suzuki, 1999c; } \\ \text { " } & \text { " } & \text { tonecedeus Sasa et Tanaka, 2000; }\end{array}$

Paratrichocladius kyogokuprimus Sasa et Suzuki, 1998b;

$\begin{array}{ll}\text { p. } 96 & \\ \text { p. } 96, & \text { Fig. } 6 \\ \text { p. } 144, & \text { Fig. } 2 \\ \text { p. } 78, & \text { Fig. } 2 \\ \text { p. } 79, & \text { Fig. } 4 \\ \text { p. } 154, & \text { Fig. } 10 \\ \text { p. } 2, & \text { Fig. } 3 \\ \text { p. } 24, & \text { Fig. } 17 \\ \text { p. } 80, & \text { Fig. } 3 \\ \text { p. } 80, & \text { Fig. } 4 \\ \text { p. } 81, & \text { Fig. } 5 \\ \text { p. } 154, & \text { Fig. } 8 \\ \text { p. } 82, & \text { Fig. } 6 \\ \text { p. } 7, & \text { Fig. } 5 \\ \text { p. } 69, & \text { Fig. } 24 \\ \text { p. } 145, & \text { Fig. } 4\end{array}$

* Note: This species was described in the original paper without giving the subgenus name, and is here classified into the above subgenus since setae on abdominal tergites are rather irregularly distributed.

Eukiefferiella gunmaquarta Sasa et Tanaka, 1998;

\begin{tabular}{ll} 
p. 2, & Fig. 2 \\
p. 80, & Fig. 5 \\
p. 86, & Fig. 13 \\
p. 97, & Fig. 7 \\
p. 99, & Fig. 8 \\
p. 84, & Fig. 9 \\
p. 85, & Fig. 11 \\
p. 85, & Fig. 12 \\
p. 86, & Fig. 13 \\
p. 87, & Fig. 14 \\
p. 88, & Fig. 15 \\
p. 8, & Fig. 6 \\
p. 71, & Fig. 26 \\
p. 71, & Fig. 27 \\
p. 72, & Fig. 28 \\
p. 73, & Fig. 29 \\
p. 19, & Fig. 30 \\
p. 20, & Fig. 31 \\
p. 20, & Fig. 32 \\
p. 2, & Fig. 4 \\
p. 3, & Fig. 5 \\
p. 158, & Fig. 11 \\
p. 88, & Fig. 16 \\
p. 89, & Fig. 17 \\
p. 91, & Fig. 19 \\
p. 92, & Fig. 21 \\
p. 21, & Fig. 33 \\
p. 93, & Fig. 22 \\
p. 77, & Fig. 34 \\
p. 78, & Fig. 35 \\
p. 78, & Fig. 36 \\
& \\
\hline
\end{tabular}

Hydrobaenus tusimolemeus Sasa et Suzuki, 1999b; uresicedea Sasa et Suzuki, 1998a; sikimiensis jokasexta Sasa et Ogata, 1999; jokaseptima " tusimofegea Sasa et Suzuki, 1999b; tusimogehea " tusimoheia "1 tusimoijea " tusimoijekea " isigaefeus Sasa et Suzuki, 2000a; yakumenea Sasa et Suzuki, 2000b; yakuneoa

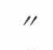

Orthocladius (Euorthocladius) tusimoopeus Sasa et Suzuki, 1999b;

Orthocladius (Orthocladius) tusimopequeus Sasa et Suzuki, 1999b;

" ( " ) sp. "yakuteuus" Sasa et Suzuki, 2000b;

Psectrocladius (Monopsectrocladius) tusimoquereus Sasa et Suzuki, 1999b;

Psectrocladius (Monopsectrocladius) yakuuveus Sasa et Suzuki, 2000b; 


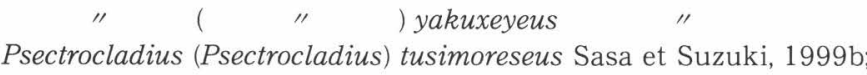
p. 79 ,
Fig. 37
p. 94 ,
Fig. 23
p. 95, Fig. 24
p. 160, Fig. 15
p. 161, Fig. 16

tusimoseteus Sasa et Suzuki, 1999b;

" " gotoheius Sasa et Suzuki, 2000c;

Setukoyusurika Sasa et Suzuki, 2000c

Setukoyusurika gotoijeus Sasa et Suzuki, 2000c;

Tribe Metriocnemini, subfamily Orthocladiinae

Apometricnemus japonicus Kobayashi et Suzuki, 1999b;

Chaetocladius perennis (Meigen, 1830), new record in Sasa and Tanaka, 1998;

p. 69

Figs. 1-9

Epoicocladius kibaprimus Sasa et Sumita, 1998;

" $\quad$ siratoriprimus Sasa et Suzuki, 1998a;
" seiryuopeus Sasa, Suzuki et Sakai, 1998b;

Heterotrissocladius sp. "tusimoteuus", in Sasa and Suzuki, 1999b;

Ikiprimus Sasa et Suzuki, 1999c;

Ikiprimus ikiijeus Sasa et Suzuki, 1999c;

Ikisecundus Sasa et Suzuki, 1999c;

Ikisecundus ikijekeus Sasa et Suzuki, 1999c;

Limnophyes siratorisecundus Sasa et Suzuki, 1998a;

jokaoctavus Sasa et Ogata, 1999;

" seiryuijeus Sasa et Sakai, 1999b;

ikikeleus Sasa et Suzuki, 1999c;

p. 4 ,

Fig. 4

p. 72 ,

Fig. 1

p. 75 ,

Fig. 1

p. 115 ,

Fig. 16

p. 96 ,

p. 157

p. 157 ,

Fig. 25

p. 158

Fig. 13

p. 158 ,

p. 76 ,

Fig. 14

p. 101,

Fig. 2

p. 109 ,

p. 159 ,

" ikilemeus "

ikimeneus

" $\quad$ isigafegeus Sasa et Suzuki, 2000a;

" $\quad$ isigafegeus Sasa et Suzuki, 2000a;

p. 159 ,

Fig. 9

p. 160 ,

p. 8 ,

p. 23 ,

Fig. 10

" yakyabeus Sasa et Suzuki, 2000b;

p. 81 ,

Fig. 15

" yakybeceus "

" yakycedeus "

" yakydeeus "

p. 81 ,

p. 82 ,

Fig. 16

" yakyefeus

p. 83 ,

Fig. 17

Metriocnemus (Metriocnemus) siratoritertius Sasa et Suzuki, 2000b;

Metriocnemus ( " " ) tusimouveus Sasa et Suzuki, 1999b;

p. 84 ,

p. 77 ,

Fig. 7

$\begin{array}{lll}\prime \prime & (1 & \text { ) ikineous Sasa et Suzuki, 1999c; } \\ \prime \prime & (1 & \text { )yakyheius Sasa et Suzuki, 2000b; } \\ \prime & (\quad \text { " } & \text { )yakyijeus, }\end{array}$

p. 97 ,

p. 163 ,

Fig. 25

Fig. 39

Fig. 40

Fig. 41

Fig. 42

Fig. 43

Fig. 3

p. $87, \quad$ Fig. 46

p. 99, Fig. 47

p. 112, Fig. 13

p. 113, Fig. 14

$\begin{array}{lll}\text { " } & \text { " } & \text { seiryumeneus " } \\ \text { " } & \text { " } & \text { tusimouveus Sasa et Suzuki, 1999b; }\end{array}$

p. $97, \quad$ Fig. 27

p. $9, \quad$ Fig. 8

p. 110 , Fig. 11

Okinawayusurika seiryujekea Sasa, Suzuki et Sakai, 1998b;

$$
\begin{aligned}
& \text { " yakyfegea Sasa et Suzuki, 2000b; } \\
& \text { " yakygehea }
\end{aligned}
$$

p. 86 ,

Fig. 44

Omuracladius, gen. nov., in Sasa et Suzuki, 1998a;

p. 87 ,

p. 87

for matsuoi (Sasa et Shimomura, 1993), recorded as Trissocladius

Parakiefferiella tusimowexeus Sasa et Suzuki, 1999b;

" yakytriangulata Sasa et Suzuki, 2000b;
" yakykelea
" yakylemea

p. 98

Fig. 45

p. 89 ,

Fig. 29

p. 90 ,

Fig. 48

p. 90 ,

Fig. 49

Paraphaenocladius tusimoxeyeus Sasa et Suzuki, 1999b;

\begin{tabular}{|c|c|}
\hline " & seiryupequea Sasa, Suzuki et Sakai, 1998b; \\
\hline " & seiryuquerea; \\
\hline " & yakymenea Sasa et Suzuki, 2000b; \\
\hline " & yakyneoa \\
\hline " & yakyopea \\
\hline " & yakypequea \\
\hline
\end{tabular}

Pseudosmittia hibaraundecima Sasa et Suzuki, 1999b;

p. $100,-$ Fig. 30

p. 27, Fig. B2

p. 117, Fig. 19

p. 118 , Fig. 20

p. 92 , Fig. 52

p. 93, Fig. 53

p. 94 , Fig. 54

p. $94, \quad$ Fig. 55 
Smittia gunmaquinta Sasa et Tanaka, 1998;

$\begin{array}{ll}\text { p. } 95, & \text { Fig. } 56 \\ \text { p. } 42, & \text { Fig. } 5 \\ \text { p. } 83, & \text { Fig. } 9 \\ \text { p. } 84, & \text { Fig. } 10 \\ \text { p. } 84, & \text { Fig. } 11 \\ \text { p. 120, } & \text { Fig. } 31 \\ \text { p. 121, } & \text { Fig. } 32 \\ \text { p. 101, } & \text { Fig. } 36 \\ \text { p. } 97, & \text { Fig. } 56 \\ \text { p. } 97, & \text { Fig. } 57 \\ \text { p. 104 } & \\ \text { p. 102, } & \text { Fig. } 32 \\ \text { p. 103, } & \text { Fig. } 33 \\ \text { p. 104, } & \text { Fig. } 34 \\ \text { p. 105, } & \text { Fig. } 35\end{array}$

Tribe Corynoneurini, subfamily Orthocladiinae

Toyamayusurika Sasa et Suzuki, 1999b;

Toyamayusurika tusimuabea Sasa et Suzuki, 1999b; " tusimubecea uresideea Sasa et Suzuki, 1998a; uresiefea Sasa et Suzuki, 1998a; uresifegea Sasa et Suzuki, 1998a; seiryuvewea Sasa, Suzuki et Sakai, 1998b; seiryuwexea Sasa, Suzuki et Sakai, 1998b; tusimoyezea Sasa et Suzuki, 1999b; yakyquerea Sasa et Suzuki, 2000b; yakyresea "

Toyamayusurika tusimucedea Sasa et Suzuki, 1999b; " tusimudeea

Fig. 35

Corynoneura ginzanquinta Sasa et Suzuki, 1998b;

p. $17, \quad$ Fig. A9

" Seiryuresea Sasa, Suzuki et Sakai 1998b;

" isigaijeus Sasa et Suzuki, 2000a;

Thienemanniella tusimuefea Sasa et Suzuki, 1999b;

" tusimufegea "
" $\quad$ gakusetea Sasa et Suzuki, 2000b;
" $\quad$ otlida Sasa et Suzuki, 2000c;

p. 123, Fig. 22

p. $10, \quad$ Fig. 9

p. $107, \quad$ Fig. 39

p. 107, Fig. 40

p. $100, \quad$ Fig. 62

p. $163, \quad$ Fig. 18

Subfamily Diamesinae

Potthastia longimana Kieffer, 1922; p. 125, Fig. 33, new name for

Diamesa (Psilodiamesa) campestris Edwards of Tokunaga (1965)

Syndiamesa kyogokusecunda Sasa et Suzuki, 1998b;

p. 25, Fig. A18

Subfamily Podonominae

Boreochlus longicoxalsetosus Kobayashi et Suzuki, 2000;

Paraboreochlus okinawanus Kobayashi et Kuranishi, 1999;

p. 319, Figs. 1-6

p. $601, \quad$ Fig. 1

\section{Subfamily Tanypodinae}

Conchapelopia seiryusetea Sasa, Suzuki et Sakai, 1998b;

" sp. B, "seiryuteua", in Sasa, Suzuki et Sakai, 1998b;

" tusimugehea Sasa et Suzuki, 1998b;

" sukayusecunda Sasa et Suzuki, 1998b;

Paramerina tusimuheia Sasa et Suzuki, 1999b;

Rheopelopia ornata (Meigen, 1838), new record in Sasa, Suzuki et Sakai, 1998b; seiryuuvea Sasa, Suzuki et Sakai, 1998b;

Protanypus sp. "inawa", in Sasa et Suzuki, 1998b;

Yaequintus Sasa et Suzuki, 2000a;

Yaequintus irioquereus Sasa et Suzuki, 2000a;

p. $126, \quad$ Fig. 25

p. 127, Fig. 26

p. 108, Fig. 41

p. $31, \quad$ Fig. B5

p. $109, \quad$ Fig. 42

p. $129, \quad$ Fig. 28

p. 129 , Fig. 29

p. $28, \quad$ Fig. B3

p. 24

p. 24, Fig. 26

\section{ACKNOWLEDGEMENTS}

The authors are especially thankful to Mr. Tadashi Kobayashi, Miss Miyoko Takagi and Mr. Hidefumi Tanaka, for their valuable assistance in preparing this report.

\section{REFERENCES}

Almost all references to taxonomy of Chironomidae in Japan are listed in the following three monographs.

Sasa, M. 1989. Chironomidae of Japan: Checklist of species recorded, key to males and taxonomic notes. Res. Rep. NIES, No. 125, 1-177.

Sasa, M. and Kikuchi, M. 1995. Chironomidae of 
Japan. 333 pp. Univ. Tokyo Press, Tokyo.

Sasa, M. 1998. Chironomidae of Japan, 1998. 156 pp.

Kankyo Fukushi Kenkyusho, Kurobe, Toyama.

\section{References published after the above monographs}

(TPES: Abbreviation of scientific report of Toyama Prefectural Institute of Environmental Science, Toyama).

Kobayashi, T. and Kuranishi, R. 1999. The second species of the subfamily Podonominae recorded from Japan, Paraboreochlus okinawanus new species. Ruffles Bull. Zool., 47: 601-606.

Kobayashi, T. and Suzuki, H. 1999a. Harnischia ohmuraensis sp. nov. and the first record of Parachironomus monochromus (van der Wulp, 1874) from Japan. Med. Entomol. Zool., 50: 79-84.

Kobayashi, T. and Suzuki, H. 1999b. The first record of the genus Apometriocnamus Saether, 1985: $A$. japonicus sp. nov. from the Palaearctic Region (Diptera, Chironomidae). Tijdschr. Entomol., 141: 6567.

Kobayashi, T. and Suzuki, H. 2000. New Podonominae from Japan, Boreochlus longicoxalsetosus sp. n. (Diptera, Chironomidae) with a key to species of the genus. Aquat. Insects, 22: 319-324.

Sasa, M. and Ogata, K. 1999. Taxonomic studies on the chironomid midges collected from the Kurobe Municipal Sewage Treatment Plant. Med. Entomol. Zool., 50: 85-104.

Sasa, M. and Sumita, M. 1998. The chironomid species collected on the shore of Lake Kibagata with light trap. Annu. Rep. Toyama Pref. Environ. Poll. Res. Cent., 1997, 70-74.

Sasa, M., Sumita, M. and Suzuki, H. 1999. The chironomid species collected with light trap at the side of Shibayamagata Lake, Ishikawa Prefecture. Trop. Med., 41: 181-196.

Sasa, M. and Suzuki, H. 1998a. Studies on the chironomid species collected in Kyushu. TPES 1998, 75-105.

Sasa, M. and Suzuki, H. 1998b. Studies on the chironomid species collected in Hokkaido and northern Honshu. Trop. Med., 40: 9-43.

Sasa, M. and Suzuki, H. 1999a. Studies on the chironomid midges of Tsushima and Iki Islands, Western Japan. Part 1. Species of Chironominae collected on Tsushima. Trop. Med., 41: 1-53.

Sasa, M. and Suzuki, H. 1999b. Studies on the chironomid midges of Tsushima and Iki Islands, Western Japan. Part 2. Species of Orthocladiinae and
Tanypodinae collected on Tsushima. Trop. Med., 41: $75-132$.

Sasa, M. and Suzuki, H. 1999c. Studies on the chironomid midges of Tsushima and Iki Islands, Western Japan. Part 3. The Chironomid Species collected on Iki Islands. Trop. Med., 41: 143-179.

Sasa, M. and Suzuki, H. 2000a. Studies on the chironomid species collected on Ishigaki and Iriomote Islands, southwestern Japan. Trop. Med., 42: 1-37.

Sasa, M. and Suzuki, H. 2000b. Studies on the chironomid midges collected on Yakushima Islands, southwestern Japan. Trop. Med., 42: 53-134.

Sasa, M. and Suzuki, H. 2000c. Studies on the chironomids collected on Goto Islands, western Japan. Trop. Med., 42: 141-174.

Sasa, M., Suzuki, H. and Sakai, T. 1998a. Studies on the chironomid midges collected on the shore of Shimanto River in April 1998. Part 1. Description of the species of the subfamily Chironominae. Trop. Med., 40: 47-89.

Sasa, M., Suzuki, H. and Sakai, T. 1998b. Studies on the chironomid midges collected on the shore of Shimanto River in April 1998. Part 2. Description of the species of additional species belonging to Orthocladiinae, Diamesinae and Tanypodinae. Trop. Med., 40: 99-147.

Sasa, M. and Tanaka, N. 1998. Notes on the chironomid species collected in Gunma Prefecture in 1996-1997. Annu. Rep. Gunma Pref. Inst. Med. \& Environ. Sci., No. 30, 37-46.

Sasa, M. and Tanaka, N. 1999. Study on the new species of chironomids collected with light traps at the side of Ino River, Gunma Prefecture. Annu. Rep. Gunma Pref. Inst. Publ. Health \& Envir. Sci., No. 31, 38-40.

Sasa, M. and Tanaka, N. 2000. Description of new species of Chironomidae collected with light traps at the side of Tone River, Gunma Prefecture. Annu. Rep. Gunma Pref. Inst. Med. \& Envir. Sci., No. $32,38-48$.

\section{摘 要}

最近に日本から新しく記録されたユスリカの種類

$$
\begin{aligned}
& \text { 佐々 学1) 鈴木 博 }{ }^{2} \\
& \text { 1) 環境福祉研究所 } \\
& \text { （テ938-0001 黒部市荒俣 135-3） } \\
& \text { 2) 長崎大学熱帯医学研究所 } \\
& \text { （干852-8523 長崎市坂本 1-12-4） }
\end{aligned}
$$

ユスリカ科の昆虫にはその地理的分布や発生源の環境 
に応じて極めて多くの種類が見出される. 日本産のユス リカについては, 主として徳永雅明が 1932 年から 1965 年にかけて, 当時の日本のほぼ全土にわたり採集 された約 160 種を記録した. 我々も 1977 年から今日に 至る約 20 年あまりの期間に, 日本全土, および近隣諸 国にわたり，広範な地域でユスリカの採集とその分類を 行い, その中, 現在の日本領土だけからでも, 佐々, 菊 池 (1985) のモノグラフ (333 頁) に扔いて 757 種類を 記録し, その中 535 種は日本特産の新種で, 131 種のみ が諸外国ですでに記録されているむのと同種と判断され ている.さらに佐々 (1998) の二冊目のモノグラフ (156 頁）に打いては新たに 176 種, 15 属が日本産ユスリカ 科として追加された。
今回の報告は，その後 1997 年 9 月から 2000 年 8 月 にかけて出版されたいろいろな学術雑誌に掲載された 20 の文献に新たに記載された日本産ユスリカ科の種類 を, 分類学的な系列に整理したものである。これらは全 部で 241 種に及び, その結果, 日本産ユスリカの記録は 1,174 種に及んだ。このリストは今後のユスリカ科の分 類学的研究には欠くべからざる資料である.

これまで佐々, および共同研究者が, 日本とその周辺 諸国で採集し，種の記載に使用したユスリカ科のスライ ド封入標本は, 2000 年 8 月末に茨城県つくば市の国立 環境研究所に保管をお願いし，そ机に伴う文献ととあ に, 国内扔よび外国よりの来訪の研究者に公開して利用 していただけるよう, 配慮した. 